\title{
A NOTE ON MYRBERG POINTS AND ERGODICITY
}

\author{
KURT FALK*
}

\begin{abstract}
The main purpose of this note is to further clarify the strong relationship between ergodicity and Myrberg type dynamics in hyperbolic manifolds. This is achieved mainly via a new suggestive proof of the fact that the Myrberg limit set of a Kleinian group is of full Patterson measure if the geodesic flow on the associated hyperbolic manifold is ergodic with respect to the PattersonSullivan measure.
\end{abstract}

\section{Introduction}

In [8] Myrberg proved the following theorem.

SATZ. Fast alle geodätischen Linien einer Fläche negativer Krümmung sind quasiergodisch, d.h. sie haben die Eigenschaft, unbegrenzt auf der Fläche fortgesetzt jeden geodätischen Bogen mit jeder Genauigkeit zu approximieren. Genauer: Unter allen durch irgend einen beliebig gewählten Punkt gehenden geodätischen Linien sind nur diejenigen nicht quasiergodisch, deren Schnittpunkte mit einer um den betreffenden Punkt beschriebenen geschlossenen regulären Kurve einer gewissen Menge angehören deren lineares Maß gleich Null ist und welche die Mächtigkeit des Kontinuums hat.

This means that Myrberg rays (referred to as 'quasiergodic' rays), namely those geodesic rays in the surface which approximate infinitely often every geodesic segment with arbitrary accuracy, form a thick set in a certain measure theoretical sense. His theorem has been generalised for instance in [20], [9] and [2]. The most general version known to the author in the context of Kleinian groups is given in [21] and [15]:

THEOREM. The Myrberg limit set $L_{\mathrm{M}}(G)$ of a Kleinian group $G$ has full Patterson measure $\mu$ if and only if the geodesic flow on the associated hyperbolic manifold $M$ is ergodic w.r.t. the Patterson-Sullivan measure v.

\footnotetext{
* Research supported by the University of St Andrews, the Schweizer Nationalfonds No. 2061379.00 and the European Project TMR "Geometric Analysis" ACR-OFES No. UE 00.0349.

Received October 10, 2002; in revised form September 25, 2003.
} 
For brief descriptions of $\mu$ and $v$ we refer to Section 2. In this note we give a new direct proof of the fact that the ergodicity of $v$ implies that $L_{\mathrm{M}}(G)$ has full $\mu$-measure (Theorem 4.2). This is done by using the so-called BirkhoffHopf limit set $L_{\mathrm{BH}}(G)$ of $G . L_{\mathrm{BH}}(G)$ stems from Hopf's generalisation [6] [7] of Birkhoff's ergodic law which, roughly speaking, states that for an ergodic system of finite mass the time mean equals the space mean. The idea is to consider a certain countable family of measurable subsets of the sphere bundle $S M$ of $M$ and, for each of these subsets, to look at the dynamics that satisfies Hopf's ergodic law (1). These dynamics give rise to certain subsets of the limit set of $G$ which are naturally associated to the initial subsets of $S M$. $L_{\mathrm{BH}}(G)$ is the intersection of all these subsets of the limit set. The ergodicity of $v$ immediately implies that each of them has full $\mu$-measure (Lemma 4.1), hence $L_{\mathrm{BH}}(G)$ is of full $\mu$-measure. Furthermore, $L_{\mathrm{BH}}(G)$ is contained in $L_{\mathrm{M}}(G)$ (Proposition 3.5), and Theorem 4.2 thus follows.

Moreover, $L_{\mathrm{BH}}(G)$ turns out to be a proper subset of $L_{\mathrm{M}}(G)$ (Remark 3.6). Hence, we have in fact refined the afore-mentioned result [21], [15] that $L_{\mathrm{M}}(G)$ has full $\mu$-measure if and only if $v$ is ergodic. This in turn was a refinement of Sullivan's result [16] that $v$ is ergodic if and only if the radial limit set of $G$ is of full $\mu$-measure. Sullivan's result is stated in detail in Section 2.

This paper is organised as follows. In Section 2 we give some preliminaries which are required in the sequel. In Section 3 we introduce the Myrberg and the Birkhoff-Hopf limit sets and discuss some of their properties. In Section 4 we investigate the relationship between ergodicity and $L_{\mathrm{M}}(G)$.

ACKNOwLEDGEMENTS. This work was part of my $\mathrm{PhD}$ thesis written at the University of St Andrews. I thank my former supervisor Bernd O. Stratmann for his permanent encouragement and unbroken patience over the years.

\section{Preliminaries}

We use the Poincaré ball model of hyperbolic space $\mathrm{H}$ which is defined as $\left\{x \in \mathrm{R}^{k}:|x|<1\right\}, k \geq 2$, together with the metric $d(\cdot, \cdot)$ derived from $d s=2|d x| /\left(1-|x|^{2}\right)$. The sphere at infinity or boundary $\partial \mathrm{H}$ of hyperbolic space is the unit sphere in $\mathrm{R}^{k}$. In the sequel we shall refer to discrete subgroups of the group of orientation preserving isometries of $\mathrm{H}$ as Kleinian groups.

Let $L(G)$ denote the limit set of the Kleinian group $G$. A point $\xi \in L(G)$ is called a radial limit point of $G$ if there exists a ball $B$ in $\mathrm{H}$ such that for every geodesic ray $r$ with endpoint $\xi$ we have $g(B) \cap r \neq \emptyset$ for infinitely many distinct $g \in G$. The set of radial limit points is called the radial limit set and we denote it by $L_{\mathrm{r}}(G) . L_{\mathrm{r}}(G)$ is also referred to as the conical limit set of $G$.

The quotient $M=\mathrm{H} / G$ of the $k$-dimensional hyperbolic space $\mathrm{H}$ through a torsion free Kleinian group $G$, i.e. a group without elliptic elements, is a 
$k$-dimensional hyperbolic manifold. For ease of notation, we keep the notation $d$ for the projected metric on $M$.

Geodesics with both their endpoints in $L(G)$ and their projections to $M$ are called canonical. The hyperbolic convex hull in $\mathrm{H}$ of the union of all canonical geodesics viewed as subsets of $\mathrm{H}$ is called the convex hull of $L(G)$ (or the Nielsen region of $G$ ). Clearly, $G$ acts discontinuously on the convex hull of $L(G)$, and quotienting out $G$ yields the convex core of $M$. Equivalently, the convex core of $M$ is the smallest convex subset of $M$ containing all closed geodesics in $M$. A non-elementary, torsion free Kleinian group is called geometrically finite if the unit neighbourhood of the convex core has finite hyperbolic volume.

Let $S \mathrm{H}$ and $S M$ denote the sphere bundles of $\mathrm{H}$ and $M=\mathrm{H} / G$ respectively, where $G$ is a torsion free Kleinian group. $G$ acts discontinuously on $S \mathrm{H}$, hence we obtain that $S M=S \mathrm{H} / G$. The geodesic flow $\phi^{t}$ on $S \mathrm{H}$ projects onto the geodesic flow on $S M$ which we also denote by $\phi^{t}$. The forward orbit $O^{+}(v)=\left\{\phi^{t}(v): t \geq 0\right\}$ of $v \in S \mathrm{H}$ projects onto a geodesic ray $r$, in the sense that $\mathrm{O}^{+}(v)$ consists of those vectors which are tangent to $r$ and which point towards the endpoint of $r$ in $\partial \mathrm{H}$. Two geodesic rays in $\mathrm{H}$ are asymptotic if they have the same point at infinity. We can also express this by saying that the forward orbits of two vectors $v, v^{\prime} \in S \mathrm{H}$ are asymptotic if there exists a constant $c \geq 0$ such that the distance between the base points in $\mathrm{H}$ of the vectors $\phi^{t}(v)$ and $\phi^{t}\left(v^{\prime}\right)$ tends to $c$ for $t$ tending to infinity. Both notions are established in an analogous way for $M$ and $S M$ by canonical projection.

The sphere bundle $\mathrm{SH}$ can be parametrised as follows. Any vector $v \in S \mathrm{H}$ determines an oriented geodesic $l$ which is tangential to $v$. Also, any vector $v \in$ $\mathrm{SH}$ determines a geodesic ray $r$, namely the geodesic ray which corresponds to $O^{+}(v)$. Clearly, $r$ is contained in the geodesic $l$. Let $\eta \in \partial \mathrm{H}$ denote the endpoint of $r$, and let $\xi \in \partial \mathrm{H}$ denote the endpoint of $l$ which is different from $\eta$. Furthermore, let $s$ denote the signed hyperbolic distance from the base point of $v$ in $\mathrm{H}$ to the Euclidean midpoint of $l$. Thus, a unit tangent vector $v \in S \mathrm{H}$ is parametrised by a triple $(\xi, \eta, s)$, where $\xi, \eta$ are two distinct elements of $\partial \mathrm{H}$, and $s \in \mathrm{R}$.

Recall Patterson's construction [11], [12] of a probability measure $\mu$ supported on the limit set $L(G)$ of a Kleinian group $G$. If $\delta$ denotes the critical exponent of the Poincaré series $P_{s}(x):=\sum_{g \in G} e^{-s d(x, g(0))}, x \in \mathrm{H}$, associated to $G$, then this Patterson measure is $\delta$-conformal, i.e. for every $g \in G$ and any measurable $A \subset \partial \mathrm{H}$ we have $\mu(g(A))=\int_{A}\left|g^{\prime}(\xi)\right|^{\delta} d \mu(\xi)$. Here, $g^{\prime}$ denotes the conformal distortion of $g$. Note that in general $\mu$ is not unique.

Using $\mu$ and the parametrisation of $S \mathrm{H}$ given above, one defines the measure $\widetilde{v}$ on $S \mathrm{H}$ by $d \widetilde{v}(\xi, \eta, s):=d \mu(\xi) d \mu(\eta) d s /|\xi-\eta|^{2 \delta}$. Now, $G$ acts discontinuously on $\mathrm{SH}$ in the following way. For an arbitrary element $g$ of $G$, 
and the parametrisation $(\xi, \eta, s)$ of some arbitrary vector in $S \mathrm{H}$, we have $g(\xi, \eta, s)=(g(\xi), g(\eta), s+t)$. Here, $t$ denotes the signed hyperbolic distance between the image under $g$ of the Euclidean midpoint of the geodesic with endpoints $\xi$ and $\eta$, and the Euclidean midpoint of the geodesic with endpoints $g(\xi)$ and $g(\eta)$. The $\delta$-conformality of $\mu$ implies that $\widetilde{v}$ is $G$-invariant, i.e. $\widetilde{v}(g(A))=\widetilde{v}(A)$ for every $g \in G$ and every measurable $A \subset S H$. Therefore, $\widetilde{v}$ projects canonically onto the Patterson-Sullivan measure $v$ defined on the sphere bundle $S M$ of $M$. Since $\mu$ is supported on $L(G)$, it follows that the support of $v$ is given by the set of all $v \in S M$ which are tangential to some canonical geodesic. Sullivan [16] generalised a method of Hopf [6], [7] in order to obtain the following result (see also [14]).

THEOREM. The following conditions are equivalent.

(i) $\mu\left(L_{\mathrm{r}}(G)\right)=1$;

(ii) the product action of $G$ on $(\partial \mathrm{H} \times \partial \mathrm{H}) \backslash\{$ diag. $\}$ is ergodic w.r.t. the product measure $\mu \times \mu$ (i.e. for any invariant set under this action, either the set itself or its complement is of zero $\mu \times \mu$-measure);

(iii) $\phi^{t}$ is ergodic w.r.t. $v$ (i.e. for any flow invariant subset of $S M$, either the set or its complement is of zero $v$-measure);

(iv) $G$ is of $\delta$-divergence type (i.e. $P_{\delta}(x)=\infty$ ).

Furthermore, if these three conditions are valid, then $\mu$ is unique.

The ergodicity of $\phi^{t}$ w.r.t. $v$ can be expressed via Hopf's version of the Birkhoff Ergodic Theorem:

THEOREM. If $h, h_{1}$ are integrable functions on $M$ such that $h_{1}>0$ and $\lim _{t \rightarrow \infty} \int_{0}^{t} h_{1}\left(\phi^{s} v\right) d s=\infty$ for $v$-almost every $v \in S M$, then

$$
\lim _{t \rightarrow \infty} \frac{\int_{0}^{t} h\left(\phi^{s} v\right) d s}{\int_{0}^{t} h_{1}\left(\phi^{s} v\right) d s}=\frac{\int_{S M} h d v}{\int_{S M} h_{1} d v}
$$

for v-almost all $v \in S M$.

Now, a central point in this situation is that there exists an integrable function $h_{1}$ with the properties required above (see [10] Theorem 7.2.10 or [16] Proposition 13). For the rest of this paper we fix such a function $h_{1}$. If $G$ is geometrically finite, then $v$ is a finite measure and all four afore-mentioned conditions are true [18]. However, there are classes of examples of geometrically infinite groups, for which these conditions are also known to hold true (see for instance [19], [17], [13] or [1]). 


\section{Myrberg and Birkhoff-Hopf limit sets}

Roughly speaking, a geodesic ray in a hyperbolic manifold $M$ is called Myrberg if it approximates with arbitrary accuracy and infinitely often every finite segment of any canonical geodesic. If we identify $\partial \mathrm{H}$ with the set of unit tangent vectors based at some fixed point in $M$, then Myrberg rays determine a certain subset of the limit set, which will be referred to as the Myrberg limit set. We remark that our definition of Myrberg rays is in the tradition of [8] and [15]. The points of view for instance in [2] or [21] are different but equivalent to ours.

As before, $G$ denotes a non-elementary, torsion free Kleinian group and $M=\mathrm{H} / G$ the associated hyperbolic manifold. Let $u$ be a geodesic segment with endpoints $x, y \in \mathrm{H}$, and consider some $\varepsilon>0$. The $\varepsilon$-flow tube $T_{\varepsilon}(u)$ associated to $u$ consists of all vectors $v \in S \mathrm{H}$ which are tangential to some geodesic segment $u^{\prime}$ with endpoints $x^{\prime}, y^{\prime} \in \mathrm{H}$ such that $x^{\prime}$ and $y^{\prime}$ project orthogonally onto $x$ and $y$ respectively, and such that $d\left(x, x^{\prime}\right) \leq \varepsilon$ and $d\left(y, y^{\prime}\right) \leq \varepsilon$. Keeping the same notation we can project such flow tubes in a canonical way onto $S M$, where they may have selfintersections.

Let $r$ be a geodesic ray in $M$ with initial vector $v \in S M$, i.e. $O^{+}(v)$ projects to $r$. Then $r$ is called a Myrberg ray if, for every $\varepsilon>0$, and every geodesic segment $u$ on any canonical geodesic in $M$, there exists a sequence of positive real numbers $t_{n} \nearrow \infty$ such that $\phi^{t_{n}}(v) \in T_{\varepsilon}(u)$ for all $n$. Also, we say that a geodesic ray in $\mathrm{H}$ is Myrberg if it projects onto a Myrberg ray in $M$. The next two lemmata give useful characterisations of Myrberg rays. The proofs are straightforward.

Lemma 3.1. Let $r$ be a geodesic ray with initial vector $v \in S M$. Then, $r$ is Myrberg if and only if the set of accumulation points of $O^{+}(v)$ in $S M$ is equal to the support of $v$, i.e. the set of all vectors in $S M$ which are tangential to some canonical geodesic of $M$.

Lemma 3.2. A geodesic ray $r$ in $\mathrm{H}$ is Myrberg if and only if for every $\varepsilon>0$ and for every geodesic segment $u$ on any canonical geodesic, there exists a sequence $\left(g_{n}\right)$ of pairwise distinct elements of $G$ such that, for all $n \in \mathrm{N}$, each point on $\left(g_{n}\right)$ ist at most $\varepsilon$ away from $r$, and such that the images of the centre of $u$ under the elements of the sequence $\left(g_{n}\right)$ converge in the Euclidean metric to the endpoint of $r$ in $\partial \mathrm{H}$.

Clearly, every ray asymptotic to some Myrberg ray is also Myrberg, which enables us to define Myrberg limit points. A point in $\partial \mathrm{H}$ is called a Myrberg limit point of $G$ if it is the endpoint of some Myrberg ray of $G$. The set of Myrberg limit points of $G$ is denoted by $L_{\mathrm{M}}(G)$. Obviously, $L_{\mathrm{M}}(G)$ is a $G$ invariant subset of $L_{\mathrm{r}}(G)$. Roughly speaking, Myrberg limit points describe 
arbitrarily fine recurrence, rather than 'coarse' recurrent dynamical behaviour given by radial limit points.

Remark 3.3. Note that $L_{\mathrm{M}}(G) \neq \varnothing$ for every non-elementary Kleinian group $G$. A proof for groups of the first kind, i.e. for which $L(G)=\partial \mathrm{H}$, can be found in [10] (see Theorem 2.2.2) where Myrberg points are called line transitive points. It is straightforward to extend this method to the general case.

Given some integrable function $h$ on $S M$, if (1) holds for a vector $v \in S M$, then it holds of course for any other vector in $O^{+}(v)$. We call a point $\xi \in L(G)$ generic w.r.t. $h$ if there exists a geodesic ray $r$ with endpoint $\xi$ and initial point $z \in \mathrm{H}$, such that the following holds. The vector $\widetilde{v} \in S \mathrm{H}$ tangential to $r$ at $z$ and pointing towards $\xi$ projects onto a vector $v \in S M$ which satisfies (1) for the function $h$. The set of such points will be denoted by $L_{h}(G)$, and we shall refer to it as the generic limit set associated to $h$. In particular, if $h$ is the characteristic function $\chi_{A}$ of some measurable set $A$ in $S M$, then we shall denote its generic limit set by $L_{A}(G)$. Note that for each integrable function $h$ on $S M$, the generic limit set $L_{h}(G)$ is $G$-invariant.

Recall that the family of closed geodesics in $M$ is countable. Therefore, the family which consists of all lifts of closed geodesics in $M$ to $\mathrm{H}$ is also countable. On each lift $\gamma$ of every closed geodesic in $M$ consider all geodesic segments which have rational length and which are centred at points whose hyperbolic distance to some fixed point on $\gamma$ is rational. Clearly, this family of segments is countable. Therefore, the family $\mathscr{T}$ of all $\varepsilon_{n}$-flow tubes associated to these segments is countable as well, where $\left(\varepsilon_{n}\right)$ denotes the sequence of positive rational numbers. For each tube $T \in \mathscr{T}$ we have a generic limit set $L_{T}(G)$ associated to its characteristic function which allows us to define the Birkhoff-Hopf limit set of $G$ by

$$
L_{\mathrm{BH}}(G):=\bigcap_{T \in \mathscr{T}} L_{T}(G) .
$$

Remark 3.4. It is not difficult to see that for every $\varepsilon>0$ and each geodesic segment $u$ on some canonical geodesic of $M$ we have $L_{\mathrm{BH}}(G) \subset L_{T_{\varepsilon}(u)}(G)$.

We shall now see that, as one would expect, the Birkhoff-Hopf limit set is contained in the Myrberg limit set of $G$. This is the crucial observation required for the proof of Theorem 4.2.

Proposition 3.5. For every non-elementary Kleinian group $G$ we have that $L_{\mathrm{BH}}(G) \subset L_{\mathrm{M}}(G)$.

Proof. Let $\xi \in L_{\mathrm{BH}}(G)$ and a geodesic ray $r$ with endpoint $\xi$ be arbitrarily chosen, and consider some $\varepsilon>0$ and some geodesic segment $u$ on an arbitrary 
canonical geodesic. Project this situation onto $M$, and keep the notations for simplicity.

We know that the set of lifts of all closed geodesics in $M$ is dense in the set of geodesics with both their endpoints in the limit set. More precisely, for any pair of disjoint open subsets $O_{1}$ and $O_{2}$ of $\partial \mathrm{H}$ both meeting the limit set of $G$, there exists a lift of a closed geodesic of $M$ which has one endpoint in $O_{1}$ and the other endpoint in $O_{2}$ (see e.g. [4] p. 97). This together with the definition of $\mathscr{T}$ implies that there exists $T \in \mathscr{T}$ which is properly contained in $T_{\varepsilon}(u)$, in the sense that $T \subset T_{\varepsilon}(u)$ and the topological boundaries of the two tubes do not have common points.

Now, since $\xi \in L_{\mathrm{BH}}(G)$, we also know that $\xi \in L_{T}(G)$. Let $r_{T}$ be the geodesic ray that gives $\xi \in L_{T}(G)$, and consider a vector $v_{T} \in S \mathrm{H}$ which is tangential to $r_{T}$ and which points towards $\xi$. Project this situation onto $M$, and keep the notations $r_{T}$ and $v_{T}$ for simplicity. We thus have that (1) holds for $v_{T}$ and for the characteristic function of $T$. (Note that each flow tube in $\mathscr{T}$ has strictly positive $v$-measure.)

Finally, by (1) and since $\lim _{t \rightarrow \infty} \int_{0}^{t} h_{1}\left(\phi^{s} v_{T}\right) d s=\infty$, it follows that $O^{+}\left(v_{T}\right)$ visits $T$, and thus $T_{\varepsilon}(u)$, infinitely often. Since $r$ and $r_{T}$ are asymptotic, and since $T$ is properly contained in $T_{\varepsilon}(u)$, the same holds for some vector tangential to $r$ and pointing towards $\xi$. Hence, by the arbitrary choice of $\varepsilon$ and $u$, we conclude that $r$ is a Myrberg ray.

Remark 3.6. In general $L_{\mathrm{BH}}(G)$ is a proper subset of $L_{\mathrm{M}}(G)$. In the case of finitely generated Schottky groups this can be seen as follows. First note that Artin [3] had already observed the occurence of Myrberg limit points (referred to as 'quasiergodic') by establishing the connection between continued fractions and the dynamics on the modular surface.

In this spirit, we shall employ one of various existing procedures for coding the dynamics on a hyperbolic manifold in order to construct certain Myrberg points which cannot be generic for a wide class of flow tubes. Let $G$ be a finitely generated Schottky group with generating set $\mathscr{A}$. By embedding the associated Cayley graph into $\mathrm{H}$ (see for instance [5]), it is not difficult to see that limit points correspond to reduced infinite words of the form $a_{0} a_{1} a_{2} \ldots$ with $a_{i} \in \mathscr{A}$.

Now, using the definition of the Cayley graph, which in our case is a tree, Lemma 3.2 and some elementary observations in hyperbolic geometry, one can show that there is a bijection from $L_{\mathrm{M}}(G)$ to the set of all infinite words which contain every finite word in the alphabet $\mathscr{A}$ at least once.

We construct a point $\xi \in L_{\mathrm{M}}(G) \backslash L_{\mathrm{BH}}(G)$ in the following way. First, since $\mathscr{A}$ is finite, there exists a listing $\omega_{1}, \omega_{2}, \omega_{3}, \ldots$ of all finite words in $\mathscr{A}$. Next, choose some letter $a \in \mathscr{A}$ and let $\alpha_{k}$ denote the word $a \ldots a$ of 
length $i_{k}$, where $i_{k} \rightarrow \infty$ is some increasing sequence of natural numbers. Finally, using concatenation, set $\omega=\omega_{1} \alpha_{1} \omega_{2} \alpha_{2} \omega_{3} \alpha_{3} \ldots$ which gives a point $\xi \in L_{\mathrm{M}}(G)$. However, if $\left(i_{k}\right)$ tends sufficiently fast to infinity, one can prove that $\xi \notin L_{T_{\varepsilon}(u)}(G)$ for $\varepsilon>0$ sufficiently small and that $u$ is not contained in the closed geodesic corresponding to the generator $a$. Hence $\xi \in L_{\mathrm{M}}(G) \backslash L_{\mathrm{BH}}(G)$.

\section{Myrberg density}

From now on we restrict our attention to such Kleinian groups $G$ and their associated hyperbolic manifolds $M$ for which the geodesic flow is ergodic w.r.t. the Patterson-Sullivan measure $v$. Employing ergodicity we find that generic limit sets associated to integrable functions have full Patterson measure. This observation will be used in the proof of Theorem 4.2.

Lemma 4.1. If the geodesic flow on $S M$ is ergodic w.r.t. $v$, then for each integrable function $h$ on $S M$, we have $\mu\left(L_{h}(G)\right)=1$.

Proof. Consider some arbitrary integrable function $h$ on $S M$. Let $E$ denote the set of all vectors $v \in S M$ such that (1) holds for $v$ and $h$. Clearly, $O^{+}(v) \subset$ $E$ for every $v \in E$. Therefore, both $E$ and its complement $E^{c}$ in $S M$ are invariant sets for the geodesic flow. It follows that the lift $\widetilde{E}$ of $E$ to $S \mathrm{H}$ and the lift $\widetilde{E}^{c}$ of $E^{c}$ to $S \mathrm{H}$ are both invariant under the geodesic flow. Hence, we have that $(\widetilde{E})^{c}=\widetilde{E}^{c}$. By definition, $L_{h}(G)$ is the projection along geodesic rays of $\widetilde{E}$ to $\partial \mathrm{H}$, in the sense that every $v \in E$ determines a geodesic ray with endpoint in $L_{h}(G)$. Also, $\partial \mathrm{H} \backslash L_{h}(G)$ is the projection along geodesic rays of $(\widetilde{E})^{c}$ to $\partial \mathrm{H}$. Since (1) holds for all vectors in $E$, it follows that $E^{c}$ is of zero $v$-measure. Recall that the Patterson-Sullivan measure $v$ on $S M$ is the projection of the measure $\widetilde{v}$ on $S \mathrm{H}$ (see Section 2). Since $v\left(E^{c}\right)=0$, it follows that $\widetilde{v}\left(\widetilde{E^{c}}\right)=\widetilde{v}\left((\widetilde{E})^{c}\right)=0$. Now, using the definition of $\widetilde{v}$, we obtain $\mu\left(\partial \mathrm{H} \backslash L_{h}(G)\right)=0$.

The following theorem relates Myrberg limit points to the phenomenon of ergodicity. The proof of this theorem in [15] mainly uses the ergodicity of the product action of $G$ on $(L(G) \times L(G)) \backslash\{$ diag. $\}$ and was inspired by Tsuji's proof [20] of Myrberg's result. We give an alternative proof which makes direct use of Hopf's Ergodic Theorem when applied to the geodesic flow on $M$.

Theorem 4.2. If the geodesic flow on SM is ergodic w.r.t. the PattersonSullivan measure $v$, then $\mu\left(L_{\mathrm{M}}(G)\right)=1$.

Proof. By Lemma 4.1 we know that the family $\left\{L_{T}(G): T \in \mathscr{T}\right\}$ consists of countably many sets of full Patterson measure. Hence, by definition of $L_{\mathrm{BH}}(G)$, we have $\mu\left(L_{\mathrm{BH}}(G)\right)=1$. The theorem now follows, since Proposition 3.5 states that $L_{\mathrm{BH}}(G)$ is contained in $L_{\mathrm{M}}(G)$. 
REMARK 4.3. Note that the converse of the assertion in Theorem 4.2 also holds. This can be seen mainly by using the facts that $L_{\mathrm{M}}(G) \subset L_{\mathrm{r}}(G)$, and that if $L_{\mathrm{r}}(G)$ has full Patterson measure, then the geodesic flow on $M$ is ergodic w.r.t. $v$ (see [16]).

REMARK 4.4. In fact we have refined the main result of [15], namely that $v$ is ergodic if and only if $\mu\left(L_{\mathrm{M}}(G)\right)=1$, to obtain that $v$ is ergodic if and only if $\mu\left(L_{\mathrm{BH}}(G)\right)=1$.

\section{REFERENCES}

1. Aaronson, J., and Sullivan, D., Rational ergodicity of geodesic flows, Ergodic Theory Dynam. Systems 4 (1984), no. 2, 165-178.

2. Agard, S., A geometric proof of Mostow's rigidity theorem for groups of divergence type, Acta Math. 151 (1983), no. 3-4, 231-252.

3. Artin, E., Ein mechanisches System mit quasiergodischen Bahnen, The collected papers of Emil Artin, Addison-Wesley, 1965, pp. 499-504.

4. Beardon, A. F., The Geometry of Discrete Groups, Springer, 1983.

5. Cannon, J. W., The combinatorial structure of cocompact discrete hyperbolic groups, Geom. Dedicata 16 (1984), 123-148.

6. Hopf, E., Fuchsian groups and ergodic theory, Trans. Amer. Math. Soc. 39 (1936), 299-314.

7. Hopf, E., Ergodic theory and the geodesic flow on surfaces of constant negative curvature, Bull. Amer. Math. Soc. 77 (1971), no. 6, 863-877.

8. Myrberg, P. J., Ein Approximationssatz fur die Fuchsschen Gruppen, Acta Math. 57 (1931), 389-409.

9. Nakanishi, T., On P. J. Myrberg's approximation theorem for some Kleinian groups, J. Math. Kyoto Univ. 25 (1985), no. 3, 405-419.

10. Nicholls, P. J., The Ergodic Theory of Discrete Groups, Cambridge University Press, 1989.

11. Patterson, S. J., The limit set of a Fuchsian group, Acta Math. 136 (1976), 241-273.

12. Patterson, S. J., Lectures On Limit Sets Of Kleinian Groups, Analytical and Geometric Aspects of Hyperbolic Space (D. B. A. Epstein, ed.), Cambridge University Press, 1987, pp. 281323.

13. Rees, M., Checking ergodicity of some geodesic flows with infinite Gibbs measure, Ergodic Theory Dynam. Systems 1 (1981), 107-133.

14. Roblin, T., Sur l'ergodicité rationelle et les propriétés ergodiques du flot géodésique dans les variétés hyperboliques, Ergodic Theory Dynam. Systems 20 (2000), 1785-1819.

15. Stratmann, B. O., A remark on Myrberg initial data for Kleinian groups, Geom. Dedicata 65 (1997), 257-266.

16. Sullivan, D., The density at infinity of a discrete group of hyperbolic motions, Inst. Hautes Études Sci. Publ. Math. 50 (1979), 171-202.

17. Sullivan, D., Travaux de Thurston sur les groupes quasi-Fuchsiens et les varietes hyperboliques de dimension 3 fibrees sur $S^{1}$, Séminaire Bourbaki, 32e année, Vol. 1979/80, Exp. 554, Lect. Notes Math., no. 842, Springer Verlag, 1981, pp. 196-214.

18. Sullivan, D., Entropy, Hausdorff measures old and new, and limit sets of geometrically finite Kleinian groups, Acta Math. 153 (1984), 259-277.

19. Thurston, W. P., The geometry and topology of three-manifolds, lecture notes, Princeton Univ., Princeton, NJ, 1979. 
20. Tsuji, M., Myrberg's approximation theorem for Fuchsian groups, J. Math. Soc. Japan 4 (1952), 310-312.

21. Tukia, P., The Poincaré series and the conformal measure of conical and Myrberg limit points, J. Anal. Math. 62 (1994), 241-259.

\author{
MATHEMATICAL INSTITUTE \\ UNIVERSITY OF BERN \\ SIDLERSTRASSE 5 \\ CH-3012 BERN \\ SWITZERLAND \\ E-mail: kurt.falk@math-stat.unibe.ch
}

